Ciencia e $\quad 2020$

\title{
Tratamiento de agua lluvia con fines de consumo humano*
}

\author{
Diana Margarita Hernández Avilés ${ }^{a}$ - Tatiana R. Chaparrob
}

Resumen: El agua lluvia es un recurso natural renovable que posee bajos índices de contaminación. En zonas rurales, el acceso al agua potable puede convertirse en una actividad poco factible. Por esta razón, la implementación de un sistema autosostenible donde el agua lluvia pueda ser tratada surge como una alternativa para evitar problemas relacionados con el estrés hídrico y la salud pública. Con base en lo anterior, el objetivo de esta investigación consistió en determinar los tratamientos necesarios para que el agua lluvia sea apta para diferentes fines de reutilización, entre estos el consumo humano. Los resultados sugieren que el filtro de carbón natural y la desinfección solar son las alternativas que presentan una mayor remoción de los contaminantes evaluados.

Palabras clave: desinfección solar; filtración; reúso; tratamientos descentralizados

Recibido: 17/12/2019. Aceptado: 17/04/2020

Disponible en línea: 09/12/2020

Cómo citar: Cómo citar: D. M. Hernández Avilés y T. Chaparro, «Tratamiento de agua lluvia con fines de consumo humano», Cien.Ing.Neogranadina, vol. 30, n.², ago. 2020.

* Artículo de investigación.

a Ingeniera civil, especialista en Recursos Hidráulicos y Medio Ambiente. Docente, Universidad Militar Nueva Granada, Bogotá, Colombia. Correo electrónico: diana.hernandez@unimilitar.edu.co

b Ingeniera civil, doctora en Ingeniería Hidráulica y Saneamiento. Docente, Universidad Militar Nueva Granada, Bogotá, Colombia. Correo electrónico: adela.rodriguez@unimilitar.edu.co 


\section{Rainwater treatment for human consumption purposes}

Abstract: Rainwater is a renewable natural resource that has low contamination indexes. In rural areas, access to potable water can become an activity that is not very feasible. It is for this reason that the implementation of a self-sustainable system where rainwater can be treated comes as an alternative to avoid problems related to hydric stress and public health. Based on the above, the objective of this research was to determine the treatments necessary for rainwater to be suitable for different reutilization purposes, being human consumption one of them. Results suggest that natural carbon filters and solar disinfection are the alternatives with the highest elimination of the contaminants evaluated.

Keywords: Solar disinfection; filtering ; reutilization; decentralized treatments 


\section{Introducción}

El suministro de agua potable en el mundo se ha convertido en un objetivo importante para varios gobiernos e instituciones que aspiran a que las comunidades puedan desarrollarse adecuadamente en el sector económico y social. Uno de los problemas más comunes y complicados que enfrentan las comunidades rurales alrededor del mundo tiene que ver con las inadecuadas condiciones para acceder a un agua potable, circunstancias que se agudizan con la disminución gradual del recurso hídrico y el consecuente incremento de los costos de consecución de agua potable [1]. La disponibilidad del agua potable en el mundo es muy variada e incluso está directamente relacionada con la pobreza y con la incapacidad de los gobiernos para financiar proyectos de saneamiento; así, regiones como África, Asia, Latinoamérica y el Caribe presentan alrededor de mil millones de personas en zonas rurales que no tienen acceso a fuentes mejoradas de agua [2]. El problema de no poder acceder a un adecuado suministro de agua potable se agudiza cuando este viene acompañado de largos periodos de sequías [3] y de la contaminación de las fuentes hídricas provocada por los vertimientos incontrolados de aguas residuales domésticas e industriales, así como por la mala disposición de residuos sólidos.

De acuerdo con la Organización Mundial de la Salud (OMs), 2100 millones de personas alrededor del mundo no tienen acceso al agua [4]. Las fuentes de agua contaminadas pueden ocasionar problemas de salud pública, debido a la propagación de enfermedades de origen hídrico, indicando que se hace necesario el uso de tratamientos eficaces para su adecuación y posterior consumo. La necesidad de ofrecerles a las personas provenientes de comunidades rurales las mejores condiciones de vida ha hecho que la comunidad científica se preocupe por considerar la implementación de varias alternativas que puedan solucionar los problemas de salubridad pública y de cuidado del ambiente. Una de las iniciativas que se ha planteado con mucha fuerza en varios países ha sido la recolección del agua lluvia, una práctica que se ha vuelto muy exitosa y de gran ayuda para suplir las necesidades domésticas y agrícolas, e.g., en Namibia [5], Kenia [6], Corea [7] y Berlín [8].

El agua lluvia puede convertirse en una fuente de agua no convencional, debido a que no existe una vinculación directa con otro tipo de suministro de agua potable. A pesar de ello, el agua lluvia puede considerarse de buena calidad, siempre y cuando se tengan los cuidados necesarios durante su captación y almacenamiento [9].

Para solucionar lo anterior, existen manuales para las buenas prácticas de captación, recolección y tratamiento del agua lluvia en zonas rurales mediante sistemas descentralizados, como se ve en la Figura 1. El uso de agua lluvia para consumo humano no tiene precedentes, debido a que la mayoría de las investigaciones que tratan este tema usan el agua lluvia tratada o sin tratamiento para usos agrícolas o productivos, sanitarios, protección de cuencas, preservación de ecosistemas, conservación de suelos y recarga de acuíferos.

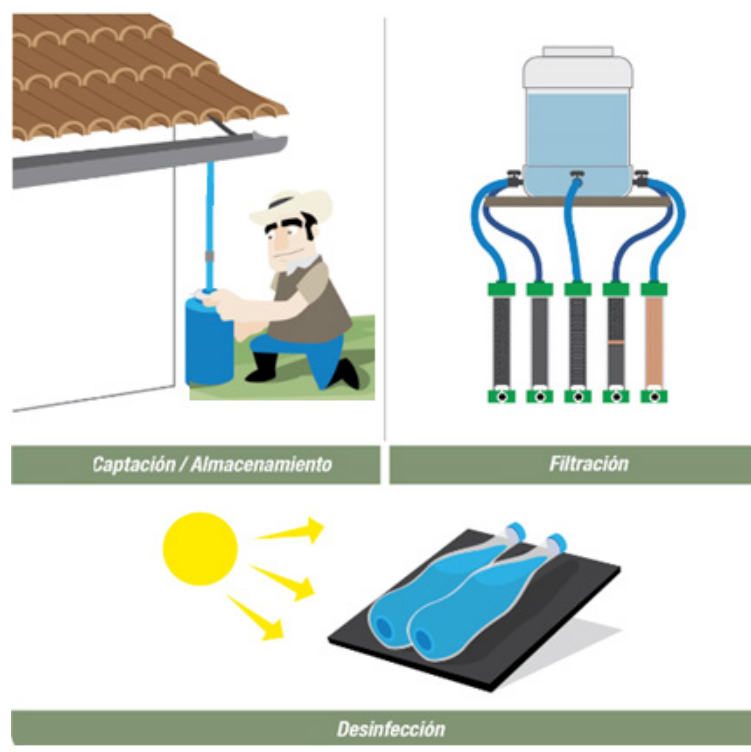

Figura 1. Sistema descentralizado de captación y tratamiento de agua lluvia en zonas rurales.

Fuente: Manual de Tratamiento de Aguas Lluvias [10].

En Colombia, la cobertura del suministro no convencional de agua para las zonas rurales en el 2011 era de alrededor un 72\%, según las cifras del Banco Mundial [11], por lo cual la recolección de agua lluvia es muy recurrida para realizar labores 
cotidianas como el riego de cultivos y lavado de baños, e.g., en San Andrés y Providencia y la ciudad de Manizales [12]. Para que el agua lluvia sea apta para consumo humano, es de gran importancia establecer qué tipo de tratamiento es el adecuado. Para algunos autores, la filtración puede actuar efectivamente en la remoción de aproximadamente el 99\% de las bacterias en el agua [1]. Además, la filtración puede contribuir en la remoción de partículas y contaminantes solubles, como los sólidos suspendidos totales y las grasas [13], reduce turbiedad y dureza [14], carbón orgánico disuelto y color [15], y remueve algunos nutrientes como nitritos y nitratos [16]. Sin embargo, aunque la filtración resulte ser un buen tratamiento, es necesario que se complemente con el proceso de la desinfección, de modo que se pueda brindar un $100 \%$ de seguridad bacteriológica a la comunidad. En ese sentido, este trabajo tiene como objetivo determinar cuál es el tratamiento más adecuado para que el agua lluvia recolectada en la Universidad Militar Nueva Granada, Bogotá, Colombia, sea apta para consumo humano.

\section{Material y métodos}

\section{Diseño experimental}

El desarrollo experimental se llevó a cabo en tres etapas. En primer lugar, la recolección y caracterización del agua lluvia en diferentes periodos de almacenamiento, esto es, el agua sin almacenar, a las 48 horas y finalmente a las 96 horas; posteriormente, la aplicación y selección de un tratamiento físico de filtrado, y finalmente, la aplicación de la desinfección utilizando la técnica Sodis (Solar
Disinfection) al tratamiento que haya mostrado la mejor efectividad en la remoción de los constituyentes físicos y químicos.

\section{Área de estudio}

El sitio de muestreo se ubica en la Universidad Militar Nueva Granada, Sede Calle 100, localizada en la zona norte de Bogotá, Colombia. La ciudad de Bogotá cuenta con una altitud de 2.640 m.s.n.m., tiene un clima frío de montaña determinado por la altitud, cuyas temperaturas oscilan entre los 7 y los $18{ }^{\circ} \mathrm{C}$, con una temperatura media anual de $14{ }^{\circ} \mathrm{C}$. Las temporadas más lluviosas del año se presentan entre abril y mayo, y entre septiembre y noviembre, alcanzando los $114 \mathrm{~mm} / \mathrm{mes}$, aproximadamente.

\section{Recolección y caracterización}

El sistema de recolección consistió en una canaleta y una bajante que conducían el agua hasta un tanque de almacenamiento de 60 litros de capacidad (Figura $1 \mathrm{a}-\mathrm{b})$. En la parte superior del tanque se colocó una tela tipo muselina, que actuó como primer filtro del sistema, removiendo hojas y desechos de animales. El agua, una vez recolectada, era almacenada y caracterizada. En la Tabla 1 se observan los ensayos realizados siguiendo los métodos descritos por la American Public Health Association (A PHA) [17]. Vale la pena indicar que estos parámetros son los establecidos por la Resolución 2115 de 2007 del Ministerio de Protección Social y del Ministerio de Ambiente, vivienda y Desarrollo Territorial de Colombia, para determinar si el agua puede ser utilizada con fines de consumo humano.

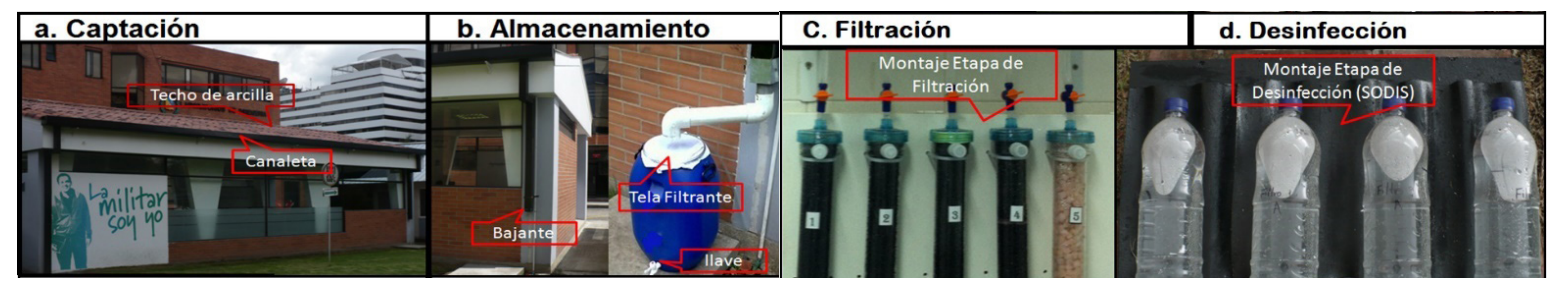

Figura 2. Sistema de tratamiento de agua lluvia que consta de un área de captación (a), un tanque de recolección y almacenamiento (b), un sistema de filtros (c) y una técnica de desinfección (d).

Fuente: elaboración propia. 
En la etapa de caracterización se evaluaron 16 muestras de agua lluvia, de las cuales 9 muestras se utilizaron para evaluar el efecto del almacenamiento durante los meses de abril hasta octubre. La siguiente etapa consistió en la selección de un tratamiento físico. En este caso se evaluaron 5 muestras y se analizaron los parámetros de la Tabla 1. Finalmente, en la etapa de desinfección se evaluó la inactivación de Escherichia coli (E. coli) para 5 muestras del agua lluvia previamente tratada en el proceso físico. Todos los ensayos de la Tabla 1 fueron realizados por duplicado y analizados utilizando estadística descriptiva en el software Origin 8.2 Pro.

\section{Sistema de filtración}

Los filtros fueron hechos en acrílico, con diámetro de $42 \mathrm{~mm}$, largo de $250 \mathrm{~mm}$, lo que resulta en una relación largo/diámetro L/D de 5,95. En el interior de cada uno de ellos se colocó primero la espuma con espesor de $32 \mathrm{~mm}$; luego el material filtrante, que se compacta en 3 capas hasta alcanzar una altura de $218 \mathrm{~mm}$, y en seguida se selló herméticamente con la tapa. Todos los filtros fueron caracterizados en laboratorio y se obtuvieron diferentes cantidades de material, propiedades físicas y tiempos de detención hidráulica que se pueden observar en la Tabla 2. Con el fin de comparar el desempeño de cada uno de los filtros se evaluaron nuevamente los parámetros de calidad mencionados en la Tabla 1.
Tabla 1. Parámetros y métodos de análisis

\begin{tabular}{|c|c|}
\hline Parámetros & Standard Methods \\
\hline $\mathrm{DB}_{05}$ & $5210 \mathrm{~B}$ \\
\hline DQO & $5220 \mathrm{C}$ \\
\hline Color (VIS436) & $2120 \mathrm{C}$ \\
\hline $\mathrm{pH}$ & $4500 \mathrm{H}+$ \\
\hline Turbiedad & $2130 B$ \\
\hline Sólidos totales & $2540 \mathrm{~B}$ \\
\hline $\mathrm{UV}_{254}$ & 5910 \\
\hline Alcalinidad Total & $2320 \mathrm{~B}$ \\
\hline Acidez Total & $2310 B$ \\
\hline Dureza & $2340 \mathrm{~B}-\mathrm{C}$ \\
\hline Cloruros & $4500 \mathrm{Cl}-\mathrm{B}$ \\
\hline Fosfatos & $4500 \mathrm{P}-\mathrm{E}$ \\
\hline Sulfatos & $4500 \mathrm{SO}_{4}$ \\
\hline E. Coli & $9222 \mathrm{D}$ \\
\hline Conductividad Eléctrica & 2510 \\
\hline
\end{tabular}

Fuente: elaboración propia.

Tabla 2. Caracterización de los materiales filtrantes

\begin{tabular}{|c|c|c|c|c|c|}
\hline Filtro & $\begin{array}{l}\text { Peso Material } \\
(\mathrm{Kg})\end{array}$ & $\begin{array}{l}\text { Densidad } \\
\left(\mathrm{g} / \mathrm{cm}^{3}\right)\end{array}$ & Porosidad (\%) & $\begin{array}{l}\text { TDH }^{\mathrm{a}} \\
\text { (horas) }\end{array}$ & $\begin{array}{l}\text { Caudal de Operación } \\
\text { (mL/min) }\end{array}$ \\
\hline Antracita & 0.242 & 1.53 & 6.62 & 9.70 & 0.5 \\
\hline Carbón activado & 0.210 & 1.96 & 30.8 & 14.5 & 0.25 \\
\hline Carbón natural & 0.079 & 1.05 & 11.2 & 18.5 & 0.25 \\
\hline Carbón Natural+antracita & $0.047-0.103$ & N.E. ${ }^{b}$ & N.E. ${ }^{b}$ & 18.9 & 0.25 \\
\hline Espuma & 0.0065 & 0.02 & 89.0 & 21.3 & 0.24 \\
\hline
\end{tabular}

aTDH: tiempo de detención hidráulica b N.E.: no especifica.

Fuente: elaboración propia. 


\section{Resultados y discusión}

\section{Calidad del agua Iluvia recolectada}

En general la calidad inicial del agua lluvia es aceptable, sin embargo, no puede ser considerada aún como potable. Inmediatamente después de un evento de lluvia, las muestras fueron caracterizadas, donde los valores medios de $\mathrm{pH}$ fueron de $6.61 \pm 0.37$ unidades; los de conductividad, $27.7 \pm 18.0 \mu \mathrm{s} / \mathrm{cm}$; alcalinidad, $9.63 \pm 5.3 \mathrm{mg} / \mathrm{L}$; cloruros, $1.08 \pm 0.57 \mathrm{mg} / \mathrm{L}$; sulfatos, $28 \pm 18.2 \mathrm{mg} / \mathrm{L}$, y dureza, $14.1 \pm 3.12 \mathrm{mg} / \mathrm{L}$. Estos valores se encuentran dentro del límite admisible establecido en la Resolución 2115 de 2007; sin embargo, en el caso del color (54.5 \pm 48.2 UPC), turbiedad $(6.34 \pm 3.8$ UNT), fosfatos $(0.91 \pm 0.49 \mathrm{mg} / \mathrm{L})$ y E. Coli $(5.84 \pm 8.8$ UFC/100 mL), los valores superan los límites permisibles establecidos. Por otra parte, la Resolución 2115 del 2007 no especifica los valores admisibles de acidez, $\mathrm{UV}_{254}$, DQO (Demanda Química de Oxígeno), $\mathrm{DBO}_{5}$ (Demanda Biológica de Oxígeno) y sólidos totales, los cuales presentan valores medios de $0.37 \pm 0.01 \mathrm{mg} / \mathrm{L}, 0.044 \pm 0.02 \mathrm{~cm}-1,41.58 \pm 43.7$ $\mathrm{mg} / \mathrm{L}, 3.06 \pm 9.3 \mathrm{mg} / \mathrm{L}$ y $31.12 \pm 18.2 \mathrm{mg} / \mathrm{L}$, respectivamente. Los resultados de la caracterización muestran que los parámetros más críticos y los que se consideran de difícil remoción son los sulfatos, la turbiedad y el DQO. En razón de lo anterior, la utilización de materiales para utilizar como lecho filtrante que contengan minerales de carbón, como la antracita y el carbón natural, resultan ser adecuados debido a su elevada superficie de contacto y capacidad de adsorción.

La capacidad de adsorción del carbón es determinada por su estructura porosa interna, donde los poros son distribuidos homogéneamente en tamaños que se consideran pequeños, reteniendo así moléculas de difícil detección [18]. Sin embargo, la remoción de las partículas contaminantes depende de múltiples factores como: el tiempo de contacto, la dosificación de carbón, la viscosidad del fluido y las características intrínsecas del mismo carbón [19].

Tabla 3.

Calidad del agua Iluvia almacenada

\begin{tabular}{|c|c|c|c|c|c|}
\hline \multirow{2}{*}{ Parámetro } & \multirow{2}{*}{ Unidades } & \multicolumn{2}{|l|}{$48 \mathrm{~h}$} & \multicolumn{2}{|l|}{$96 \mathrm{~h}$} \\
\hline & & M1 & DE2 & M1 & DE2 \\
\hline $\mathrm{pH}$ & unidades & 6.71 & 0.13 & 6.57 & 0.28 \\
\hline Conductividad & $\mu \mathrm{s} / \mathrm{cm}$ & 13.7 & 2.27 & 13.5 & 1.95 \\
\hline Turbiedad & UNT & 7.54 & 1.33 & 2.32 & 0.66 \\
\hline Color & UPC & 16 & 7.35 & 13.5 & 8.96 \\
\hline $\mathrm{uv}_{254}$ & - & 0.024 & 0.005 & 0.022 & 0.004 \\
\hline Acidez & $\mathrm{mg} \mathrm{CaCO}_{3} / \mathrm{L}$ & 0.28 & 0.11 & 0.26 & 0.09 \\
\hline Alcalinidad & $\mathrm{mgCaCO}_{3} / \mathrm{L}$ & 12.7 & 2.41 & 14.92 & 2.18 \\
\hline DQO & $\mathrm{mg} / \mathrm{L}$ & 31.9 & 17.9 & 14.12 & 8.25 \\
\hline Fosfatos & $\mathrm{mgPO}_{4} / \mathrm{L}$ & 0.67 & 0.09 & 0.73 & 0.14 \\
\hline Cloruros & $\mathrm{mg} \mathrm{Cl} / \mathrm{L}$ & 0.92 & 0.11 & 0.49 & 0.0 \\
\hline Sulfatos & $\mathrm{mgSO}_{4} / \mathrm{L}$ & 71.4 & 11.02 & 76.7 & 4.80 \\
\hline Dureza & $\mathrm{mg} \mathrm{CaCO}_{3} / \mathrm{L}$ & 13.4 & 1.28 & 15.1 & 1.78 \\
\hline Sólidos totales & $\mathrm{mg} / \mathrm{L}$ & 23.8 & 7.98 & 16.4 & 3.7 \\
\hline E. Coli & UFC/ $100 \mathrm{~mL}$ & 1.73 & 1.33 & 2.38 & 1.86 \\
\hline
\end{tabular}

${ }^{1}$ M: Media; ${ }^{2}$ DE: desviación estándar.

Fuente: elaboración propia. 


\section{Almacenamiento del agua lluvia}

En la Tabla 3 se presentan los valores medios del agua lluvia con tiempos de almacenamiento de 48 y $96 \mathrm{~h}$. Los resultados indican que el almacenamiento, a las 48 horas y 96 horas, influye de manera notoria, por decantación, en las concentraciones de turbiedad, DQO, cloruros y solitos totales. Por tanto, se evidencia que almacenar el agua lluvia por 96 horas puede mejorar la calidad del agua, si bien existe el riesgo de presentar una mayor concentración de microrganismos y bacterias, lo cual se debe tener en cuenta para una posterior aplicación de los tratamientos seleccionados. Los tiempos de ocurrencia de eventos de lluvia influyen en la variación de los parámetros de calidad; por lo tanto, se recomienda que al implementar un sistema de recolección de agua lluvia se usen materiales que no degraden su calidad y que exista una limpieza constante en el techo, canaleta, bajante y tanque de almacenamiento.

\subsection{Selección de tecnologías}

Las siguientes etapas experimentales fueron definidas conforme a los valores medios de los parámetros de calidad obtenidos en la fase de caracterización del agua lluvia sin almacenar.

\section{Filtración}

Se implementó un sistema constituido por 5 filtros (Figura 1 c), cada uno con diferente material filtrante, a saber: antracita (1), carbón activado granular (2), carbón natural (3), una mezcla de carbón natural y antracita (4), y finalmente espuma (5). Los carbones en un sistema de filtración han resultado ser muy útiles [20], además de ser usados en las plantas de tratamiento de agua potable, debido a que las propiedades físicas de estos mismos les permiten retener compuestos orgánicos que causan color, olor y sabor indeseables; también son capaces de reducir las concentraciones de sólidos, DQO y dureza. La espuma de poliuretano ha sido utilizada como medio de soporte para la retención de biomasa en estudios realizados en reactores anaerobios para evaluar la remoción de contaminantes emergentes y compuestos no biodegradables presentes en aguas residuales, tanto domésticas como industriales, mostrando resultados promisorios [21].

En la Tabla 4 se muestran los resultados de la calidad del agua lluvia filtrada por cada uno de los filtros evaluados. El filtro 3 presenta los mejores desempeños para reducir las concentraciones de partículas y contaminantes presentes en el agua lluvia. Las eficiencias de remoción obtenidas son de $80 \%$ para color, $79 \%$ para turbiedad, $77 \%$ para cloruros, $53 \%$ para fosfatos, $69 \%$ para sulfatos, $92 \%$ para DQO y $100 \%$ para $\mathrm{DBO}_{5}$. En la literatura no se han encontrado estudios similares. En general, los estudios que hay son de utilización de agua lluvia con fines de irrigación agrícola o sobre la reutilización de las aguas grises con membranas metálicas, las cuales remueven nutrientes como nitrógeno y fosfatos en un 22 y $32 \%$ [22] y de los biofiltros conformados por suelo, arena y grava que pueden remover fósforo, nitrógeno y sólidos suspendidos en un 70\%, 85\% y 95\% [23]. El agua lluvia con fines de lavado de baños y de jardines ha sido tratada con métodos costosos y no autosostenibles, como lo exponen Kim et al., al indicar que las membranas metálicas combinadas con la aireación o la inyección de ozono pueden remover las partículas contaminantes y los microorganismos, presentado resultados para la turbiedad de $2.0 \mathrm{UNT}$, $\mathrm{pH}$ de 7.5 unidades, conductividad de $123.7 \mu \mathrm{S} / \mathrm{cm}$, fosfatos de $0.2 \mathrm{mg} / \mathrm{L}$, color 22 uPC y sin presencia de coliformes a los 10 minutos de inyectar ozono [24].

En el filtro de antracita, los parámetros que se redujeron fueron: color, turbiedad, cloruros, fosfatos, dureza, DQO, $\mathrm{DBO}_{5}$ y E. Coli. En el caso de los sulfatos y sólidos totales, estos no mostraron una reducción en su concentración; sin embargo, estos resultados son aceptables de acuerdo con la Resolución 2115 de 2007. El pH disminuyó notablemente, pasó de 6.61 unidades a 3.73 unidades. De acuerdo con Hong y Slatick, este comportamiento se presentó debido a las propiedades de la antracita como 
emisora de gran cantidad de dióxido de carbono [25]. La reacción química entre el dióxido de carbono y el agua forma un ácido débil llamado ácido carbónico, explicando de esta manera el cambio en el pH del agua. Debido a esto, si se compara con la Resolución 2115 de 2007, el pH y la alcalinidad no cumplen con los estándares vigentes.

En los filtros de 2, 4 y 5, se observa que la eficiencia de remoción de la mayoría de los parámetros estudiados es menor con respecto a los filtros de antracita y carbón natural, incluso para el filtro de carbón activado granular se observó que, en parámetros tales como cloruros, sulfatos, dureza y sólidos totales, la concentración aumentó después de la filtración. No obstante, el agua tratada en estos filtros podría ser consumida, pues de acuerdo con la Resolución 2115 de 2007 sí cumplen la norma. En estos mismos filtros, se observó que los parámetros de color, turbiedad y fosfatos no cumplen con la Resolución 2115 de 2007. Con base en esto, estos tipos de lechos filtrantes se descartan en este trabajo para continuar con la etapa de desinfección.

\section{Desinfección}

Para aplicar el proceso de desinfección se seleccionó la técnica Sodis (Figura 1d), que consiste en: una placa de madera con $15^{\circ}$ de inclinación, con el fin de mejorar la técnica del Sodis [26], sobre la cual se coloca una teja plástica. Ambas fueron recubiertas por una mezcla de pintura negra con dióxido de titanio en polvo, que actúa como agente catalizador de la desinfección [2]. Sobre la teja y la placa se colocaron 4 botellas tipo PET, cada una con un volumen de 1 l. Las botellas se expusieron a la radiación solar por un periodo de tiempo de 6 horas aproximadamente, de forma tal que se pueda garantizar una correcta inactivación de los microorganismos. Autores como McGuigan indican que periodos largos de exposición son adecuadosenzonasdondeesmayorlanubosidad [27]. La aplicación de Sodis resultó ser muy eficiente para la inactivación del Escherichia coli, registrando para todas las muestras del agua lluvia, previamente filtrada por la antracita y el carbón natural, valores de 0 UFC/100 mL. Además, con la prueba de control realizada al agua lluvia tratada con filtro de antracita y Sodis, se evidenció que el uso de Sodis disminuyó un poco más los niveles de concentración de la conductividad, turbiedad, $\mathrm{UV}_{254}$, DQO, fosfatos, sulfatos, dureza y sólidos totales, con valores de $12.5 \mu \mathrm{s} / \mathrm{cm}, 1 \mathrm{UNT}, 0.011 \mathrm{~cm}-1,2.49$ $\mathrm{mg} / \mathrm{L}, 0.17 \mathrm{mg} / \mathrm{L}, 4.61 \mathrm{mg} / \mathrm{L}, 3 \mathrm{mg} / \mathrm{L}$ y $10 \mathrm{mg} / \mathrm{L}$, respectivamente. Sin embargo, el agua lluvia tratada por esta alternativa presenta valores de $\mathrm{pH}$ que no cumplen con el rango estipulado por la Resolución 2115 del 2007, lo que dificulta su uso para la potabilización del agua lluvia.

Una vez realizada la prueba de control al agua lluvia tratada con filtro de carbón natural y Sodis, se evidenció que el Sodis, de manera similar a la anterior alternativa, generó reducciones en los parámetros de turbiedad, color, UV254, DQO, fosfatos, sulfatos y sólidos totales, con valores de $1 \mathrm{UNT}, 10$ UPC, $0.016 \mathrm{~cm}-1,0 \mathrm{mg} / \mathrm{L}, 0.26 \mathrm{mg} / \mathrm{L}, 7.79 \mathrm{mg} / \mathrm{L} \mathrm{y}$ $11.11 \mathrm{mg} / \mathrm{L}$, respectivamente; los demás parámetros de calidad se comportaron de manera estable después del proceso de desinfección. En vista de los resultados obtenidos en esta investigación, se determinó que el tratamiento de agua lluvia con el filtro de carbón natural y Sodis es el más efectivo al momento de remover la carga contaminante. El filtro de carbón natural y el Sodis resultarían ser una solución descentralizada para aquellas comunidades de bajos recursos y que se encuentran desconectadas de una red de acueducto. Por lo tanto, este tipo de solución es recomendable para desarrollar viviendas urbanas y rurales sostenibles con poca inversión en operación y mantenimiento. 
Tabla 4. Calidad del agua lluvia filtrada

\begin{tabular}{|c|c|c|c|c|c|c|c|c|c|c|c|}
\hline \multirow{2}{*}{ Parámetros } & \multirow{2}{*}{ Unidades } & \multicolumn{2}{|l|}{1} & \multicolumn{2}{|l|}{2} & \multicolumn{2}{|l|}{3} & \multicolumn{2}{|l|}{4} & \multicolumn{2}{|l|}{5} \\
\hline & & $M^{1}$ & $\mathrm{DE}^{2}$ & $M^{1}$ & $\mathrm{DE}^{2}$ & $M^{1}$ & $\mathrm{DE}^{2}$ & $M^{1}$ & $\mathrm{DE}^{2}$ & $M^{1}$ & $\mathrm{de}^{2}$ \\
\hline $\mathrm{pH}$ & unidades & 3.73 & 0.12 & 7.55 & 0.21 & 7.34 & 0.14 & 6.76 & 0.24 & 6.35 & 0.52 \\
\hline Conductividad & $\mu \mathrm{s} / \mathrm{cm}$ & 49.80 & 14.64 & 182.93 & 148.82 & 53.80 & 20.51 & 38.90 & 12.45 & 26.91 & 12.58 \\
\hline Acidez & $\mathrm{mgCaCO}_{3} / \mathrm{L}$ & 1.48 & 0.56 & 0.12 & 0.11 & 0.28 & 0.18 & 0.45 & 0.09 & 0.34 & 0.21 \\
\hline Alcalinidad & $\mathrm{mgCaCO}_{3} / \mathrm{L}$ & 0.00 & 0.00 & 20.88 & 8.13 & 22.38 & 9.60 & 9.30 & 4.51 & 7.40 & 3.83 \\
\hline Color & UPC & 8.60 & 3.21 & 12.20 & 3.03 & 10.80 & 3.42 & 19.00 & 11.11 & 18.60 & 4.77 \\
\hline Turbiedad & UNT & 1.56 & 0.22 & 2.32 & 0.51 & 1.32 & 0.31 & 4.12 & 2.65 & 1.50 & 0.00 \\
\hline Cloruros & $\mathrm{mg} \mathrm{Cl} / \mathrm{L}$ & 0.32 & 0.09 & 1.63 & 1.24 & 0.25 & 0.07 & 0.52 & 0.49 & 0.42 & 0.14 \\
\hline Fosfatos & $\mathrm{mgPO}_{4} / \mathrm{L}$ & 0.52 & 0.03 & 0.83 & 0.20 & 0.43 & 0.04 & 0.64 & 0.42 & 0.59 & 0.32 \\
\hline Sulfatos & $\mathrm{mgSO}_{4} / \mathrm{L}$ & 30.36 & 6.95 & 99.08 & 133.07 & 8.67 & 2.13 & 13.73 & 7.30 & 7.60 & 2.57 \\
\hline Dureza & $\mathrm{mgCaCO} / \mathrm{L}$ & 8.28 & 2.01 & 75.16 & 73.89 & 20.80 & 7.79 & 13.28 & 2.35 & 11.08 & 2.63 \\
\hline DQO & $\mathrm{mg} / \mathrm{L}$ & 3.50 & 7.83 & 4.98 & 11.14 & 3.50 & 6.52 & 6.25 & 8.20 & 9.82 & 12.80 \\
\hline Sólidos Totales & $\mathrm{mg} / \mathrm{L}$ & 33.35 & 12.73 & 119.15 & 114.04 & 26.15 & 18.35 & 21.08 & 10.44 & 19.77 & 8.52 \\
\hline $\mathrm{UV}_{254}$ & $\mathrm{~cm}^{-1}$ & 0.02 & 0.01 & 0.02 & 0.01 & 0.02 & 0.01 & 0.03 & 0.02 & 0.03 & 0.02 \\
\hline E.Coli & UFC / $100 \mathrm{~mL}$ & 3.20 & 4.32 & 1.40 & 2.19 & 5.40 & 8.05 & 1.20 & 2.68 & 15.60 & 33.23 \\
\hline
\end{tabular}

${ }^{1}$ M: media; ${ }^{2} D E$ : desviación estándar.

Fuente: elaboración propia.

\section{Conclusiones}

El sistema de tratamiento que mejor se desempeñó para la remoción de materia orgánica, color, sólidos y Escherichia coli fue el de filtración con carbón natural seguida de la desinfección solar. Asimismo, se observó que almacenar el agua hasta por un periodo de 96 horas sí influye en la calidad inicial, por lo cual se considera importante utilizar recipientes de almacenamiento duraderos e inertes para evitar la contaminación del agua lluvia y que no exista ninguna perturbación en el sitio de almacenamiento, y prevenir que todo material sedimentado sea resuspendido en el agua. Aunque los parámetros evaluados del agua lluvia tratada mediante el sistema seleccionado cumplen con la normatividad colombiana para consumo humano, en esta investigación no es posible afirmar si este tratamiento realiza una adecuada potabilización del agua lluvia. Por lo tanto, se recomienda incluir la evaluación de parámetros tales como nitrógeno, hongos, ensayos de toxicidad 
por metales pesados e incluso estudios de modelación de transporte de contaminantes atmosféricos que son esenciales para verificar la influencia de las nubes contaminadas en el comportamiento químico del agua lluvia.

\section{Agradecimientos}

Las autoras expresan sus agradecimientos a la Universidad Militar Nueva Granada por su apoyo financiero. Al Laboratorio de Calidad de Aguas y al Laboratorio de Saneamiento Ambiental por el apoyo técnico, y al grupo de investigación en Agua y Energía.

\section{Referencias}

[1] S. Malato, S. P. Fernández-Ibáñez, M. I. Maldonado, J. Blanco y W. Gernjak, "Decontamination and disinfection of water by solar photocatalysis: recent overview and trends", Cat. Today, vol. 14, n. ${ }^{\circ}$ 1, 2009. pp. 1-59. doi: 10.1016/j.cattod.2009.06.018.

[2] S. Gelover, L.A. Gómez, K. Reyes y Ma. Leal, “A practical demonstration of water disinfection using TiO2 films and sunlight water", Wat. Res., vol. 20, n. ${ }^{\circ} 17,2006$. pp.3274-3280. doi: 10.1016/j.watres.2006.07.006

[3] A. Mankad, "Decentralised water systems: Emotional influences on resources decision making", Env. Int., vol. 44, n. ${ }^{\circ}$ 1. pp.128-140, 2012. doi: 10.1016/j.envint.2012.01.002

[4] N. Osseiran. (2017, jul. 12). "Organización Mundial de la Salud". [Internet]. Disponible en: https://www. who.int/es/news-room/detail/12-07-2017-2-1-billionpeople-lack-safe-drinking-water-at-home-more-thantwice-as-many-lack-safe-sanitation

[5] M. Sturm, M. Zimmermann, K. Schütz, W. Urban y H. Hartung, "Rainwater harvesting as an alternative water resource in rural sites in central northern $\mathrm{Na}$ mibia", Phys. and Chem. of the Earth, parts A/B/C, vol. 34, n. ${ }^{\circ}$ 13-16, pp. 776-785, 2010. doi: 10.1016/j. pce.2009.07.004

[6] L. W. Gill y C. Price, "Preliminary observations of a continuous flow solar disinfection system for a rural community in Kenya”. Energy, vol. 35, n. ${ }^{\circ} 12$. pp. 46074611, 2010. doi: 10.1016/j.energy.2010.01.008
[7] B. McCann, "Global prospects for rainwater harvesting", Water 21, Mag. of the Int. Water Assoc., n. ${ }^{\circ}$ 10.6, pp.12-14, 2008.

[8] K.W. König, "German development provides rainwater". Water 21, Mag. of the Int. Water Assoc., n. ${ }^{\circ}$ 10.3, pp. 29, 2008.

[9] E. Wisbeck, E. K. Sandri, A. L. Soares y S. H. W. Medeiros, "Desinfecção de água de chuva por radiação ultravioleta”, Engen. Sanit. e Amb., vol. 16, n. ${ }^{\circ}$ 4, pp.337-342, 2011. doi: 10.1590/S1413-41522011000400004.

[10] D. M. Hernández, D. B. Pedraza y T. Rodriguez, Manual de tratamiento de Aguas Lluvias. Bogotá: Universidad Militar Nueva Granada, 2013. doi: 10.13140/ RG.2.2.25579.54568

[11] Banco Mundial. Datos Colombia. [Internet] En: datos. bancomundial.org/país/Colombia.

[12] M. Pacheco Montes, “Avances en la Gestión Integral del Agua Lluvia (giall): contribuciones al consumo sostenible del agua, el caso de 'Lluviatl' en México", Rev. Int. Sost., Tec. y Hum., n. o 3, pp. 39-57, 2008.

[13] H. J. Master, S. M. Kraemer, R. B. Johnston, "Achieving long-term use of solar water disinfection in Ziwbawe”. Pub. Health, vol. 127, n. ${ }^{\circ}$. pp. 92-98, 2013. doi: 10.1016/j.puhe.2012.09.001

[14] R. F. Moreira, M. L. Calijuri, I. de Castro, y A. da Fonseca, "Rainwater treatment in airports using slow sand filtration followed by chlorination: Efiiciency and costs". Resour., Conserv. and Recyc, vol. 65, pp. 124129, 2012. doi: 10.1016/j.resconrec.2012.06.001

[15] P. Singh, P. Kumar, I. Mehrotra y T. Grischek, "Impact of river bank filtration on treatment of polluted river water", Journ. of Env. Manage, vol. 91, n. ${ }^{\circ} 1-3$, pp. 227289, 2010. doi: 10.1016/j.jenvman.2009.11.013

[16] J. A. Herrera, J. A. González, O. Díaz, M. E. Aguilar, M. E. Bujalance y J. M. Doña, "Effect of stone filters in a pond wetland system treating raw wastewater from a university campus", Desalination, vol. 237, n. ${ }^{\circ} 1-3$. pp. 227-289, 2009. doi: 10.1016/j.desal.2008.01.021

[17] E. W. Rice, R. B. Baird, A. D. Eaton, Standard Methods for the Examination of Water and Wastewater (21a ed.). Washington, DC: American Public Health Association APHA, 2017.

[18] M. Ahmedna, W. E. Marshall y R. M. Rao, "Surface properties of granular activated carbons from agricultural by-products and their effects on raw sugar deco- 
lorization", Bioresource Tech., vol. 71, n. ${ }^{\circ}$ 2. pp. 103-112, 2000. doi: 10.1016/S0960-8524(99)90069-X

[19] M. Ahmedna, W. E. Marshall y R. M. Rao, "Production of granular activated carbons from select agricultural by-products and evaluation of their physical, chemical and adsorption properties", Bioresource Tech.., vol. 71, n. ${ }^{\circ}$ 2. pp. 113-123, 2011. doi: 10.1016/ S0960-8524(99)00070-X

[20] M. Rivera, V. Gómez-Serrano, P. M. Álvarez, M. C. M. Alvim-Ferraz y J. M. Dias, "Activated carbon modifications to enhance its water treatment applications: An overview", Jour. of Haz. Mat., Vol. 187, n. ${ }^{\circ} 1-3$. pp. 1-23, 2011. doi: 10.1016/j.jhazmat.2011.01.033

[21] D. Grisales, J. Ortega y T. R. Chaparro, "Remoción de la materia orgánica y toxicidad en aguas residuales hospitalarias aplicando ozono", Rev. Dyna, vol. 79, n. ${ }^{\circ}$ 173, pp. 109-115, 2012.

[22] R. H. Kim, S. Lee, J. Jeong, J. Lee y K. Kim, "Reuse of greywater and rainwater using filter media and metal membrane", Desalination, vol. 202, n. ${ }^{\circ}$ 1, 3, 5, pp. 326332, 2007. doi: 10.1016/j.desal.2005.12.071

[23] K. Bratieres, T. D. Fletcher, A. Deletic y. Zinger, "Nutrient and sediment removal by stormwater biofilters: A large scale design optimization study", Water Res., vol. 42 , n. ${ }^{\circ} 14$, pp. $3930-3940$, 2008. doi: 10.1016/j.watres.2008.06.009

[24] R. H. Kim, S. Lee y J. O. Kim, "Application of a metal membrane for rainwater utilization: filtration characteristics and membrane fouling", Desalination. Vol. 177, n. ${ }^{\circ} 1-3$. pp. 121-132, 2005. doi: 10.1016/j.desal.2004.12.004

[25] M. B. Fisher, M. Iriarte y K. L. Nelson, "Solar water disinfection (SODIS) of Escherichia coli, Enterococcus. Spp., and MS2 Coliphage: effects of additives and alternative container materials", Water Res, vol. 46, n. ${ }^{\circ}$, pp. 1745-1754, 2012. doi: 10.1016/j.watres.2011.12.048

[26] M. T. Amin y M. Y. Han, "Roof-harvested rainwater for potable purposes: application of a solar collection disinfection", Water Res., vol. 43, n. ${ }^{\circ} 20$, pp. 5225-5235, 2009. doi: 10.1016/j.watres.2009.08.041.

[27] K. G. McGuigan, R. M. Conroy, H. J. Mosler, M. Preez, E. Ubomba-Jaswa y P. Fernández-Ibáñez, "Solar Water Disinfection (Sodis): A review from bench-top to roof top", Jour. of Haz. Mat., vol. 29, pp. 235-236, 2012. doi: 10.1016/j.jhazmat.2012.07.053 